\title{
Long-term Follow-up of the QuantiFERON TB-2G Test for Active Tuberculosis Disease
}

\author{
Yoshihiro Kobashi, Tadaaki Sugiu, Yoshihiro Ohue, Keiji Mouri, Yasushi Obase, \\ Naoyuki Miyashita and Mikio Oka
}

\begin{abstract}
Objective To evaluate transitional changes in QuantiFERON TB-2G (QFT-2G) test results in the serial testing on the same patients and to reevaluate the optimal threshold of positive response of QFT-2G test as a cure of TB infection.

Methods We prospectively investigated transitional changes of QFT-2G test results in 22 patients with active tuberculosis (TB) over three years after the initiation of treatment with antituberculosis drugs. Treatment using antituberculosis drugs was performed for six months in all patients.

Results The positive rate of QFT-2G test results decreased 50\% at the treatment completion. Thereafter, although the positive rate of QFT-2G test results has been decreased $45 \%$ six months later even if treatment was finished, it decreased slightly to $41 \%$ two years later and $36 \%$ three years later. If the cut-off value was situated below 50\% (IFN- $\gamma$ level three years later/ IFN- $\gamma$ level of baseline peak value), we could judge the conversion of QFT-2G test in most cases except for two cases three years after the initiation of antituberculosis treatment through this study.

Conclusion It may be difficult to monitor markers in the cure of TB infection using QFT-2G tests. The cutoff level for a positive response on QFT-2G test may need to be reconsidered when the test is used to monitor the response of active TB to therapy.
\end{abstract}

Key words: quantiFERON TB-2G (QFT-2G), active tuberculosis (TB), antituberculosis treatment, monitoring marker

(Inter Med 47: 1957-1961, 2008)

(DOI: 10.2169/internalmedicine.47.1313)

\section{Introduction}

To date, although there have been a few reports on the performance of individual interferon- $\gamma$ release assays (IGRAs; QFT-2G test or T-SPOT.TB test) on serial testing $(1,2)$ for latent TB infection (LTBI) or active TB, there are no published data on the performance of IGRAs in serial tests in the same patient with active TB over a three-year period. It is necessary to generate evidence regarding the variability of IFN- $\gamma$ responses during serial testing over long-term follow-up, frequency of conversions and reversions, and thresholds to distinguish cure or relapse of active TB. Therefore, we prospectively evaluated transitional changes in QFT-2G test results in serial testing for three years after the initiation of antituberculosis treatment for patients with active TB and reevaluated the optimal threshold of positive response as a cure of TB infection.

Methods
Twenty-two patients were confirmed to be positive for
Mycobacterium tuberculosis (MTB) by culture of sputum,
bronchoalveolar lavage fluid, pleural fluid, peritoneal fluid,
or lymph node tissue samples who periodically consulted
the hospital for at least three years were prospectively en-
rolled starting in January 2005. This study was approved by
the institutional ethical committee at each hospital. Patients
were diagnosed at Kawasaki Medical School Hospital and
four affiliated hospitals. All patients had negative results on


Table 1. Clinical Characteristics of 22 Patients with Active Tuberculosis (TB)

\begin{tabular}{|c|c|}
\hline Clinical characteristics & Patients with active TB disease $(n=22)$ \\
\hline Age, years (Mean \pm S.D.) & $59.4 \pm 10.2$ \\
\hline Sex (Male : Female) & $17: 5$ \\
\hline Smoking history & $14(64 \%)$ \\
\hline Alcohol abuse history & $4(18 \%)$ \\
\hline Underlying disease & $16(73 \%)$ \\
\hline Respiratory disease & $7(32 \%)$ \\
\hline Healed pulmonary TB & 3 \\
\hline COPD & 2 \\
\hline Others & 2 \\
\hline Non-respiratory disease & $9(41 \%)$ \\
\hline Gastrointestinal disease & 2 \\
\hline Diabetes mellitus & 2 \\
\hline Cancer & 2 \\
\hline Cardiovascular disease & 1 \\
\hline Collagen vascular disease & 1 \\
\hline Chronic renal failure ${ }^{a}$ & 1 \\
\hline Immunosuppressive treatment ${ }^{\mathrm{b}}$ & $2(9 \%)$ \\
\hline Received BCG vaccination & $13(59 \%)$ \\
\hline Received previous TB treatment & $4(18 \%)$ \\
\hline
\end{tabular}

serological tests for HIV or an absence of obvious risk factors for HIV infection. Concerning the antituberculosis treatment, we administered four antituberculosis drugs to $22 \mathrm{pa}$ tients with active TB disease for the initial two months [isoniazid (INH), rifampicin (RFP), pyrazinamid (PZA) and ethambutol (EB) or streptomycin (SM)] and then three antituberculosis drugs for the following four months (INH, RFP, EB). These agents have been recommended by the World Health Organization (WHO). Demographic data from patients with active TB disease were collected, including any history of previous $\mathrm{TB}$ or antituberculosis treatment, other underlying diseases and administration of immunosuppressive drugs within three months before enrollment in this study.

The QFT-2G test was performed according to the manufacturer's recommendation and the test was judged according to the CDC guidelines (3). The QFT-2G test was performed for all patients before initiating the administration of antituberculosis drugs, then repeated at $6,12,18,24$ and 36 months after the initiation of antituberculosis treatment (at the completion of treatment).

The conversion rate of QFT-2G test results was calculated according to the cut-off value divided into 10 percent increments both at the completion of treatment and three years after the initiation of antituberculosis treatment. The cut-off value was defined as the percentage of IFN- $\gamma$ level at the completion of treatment (or after three years) per IFN- $\gamma$ level of baseline peak value.

The analysis consisted of student's $t$ test, for evaluating differences in means on the basis of logarithmic transformation of IFN- $\gamma$ measurements, the $\chi^{2}$ test, for testing the difference in proportions, and maximum-likelihood logistic regression, for estimating the strength of the association between the duration of antituberculosis treatment and the re- sults of whole blood IFN- $\gamma$ assay. The level of significance was $\mathrm{p}<0.05$.

\section{Results}

The final diagnosis of 22 patients consisted of pulmonary TB in 14 patients (including pulmonary tuberculoma in 1), tuberculous pleuritis in 3, tuberculous lymphangitis in 2 , pulmonary $\mathrm{TB}$ and tuberculous pleuritis in 1, miliary $\mathrm{TB}$ in 1 , and tuberculous peritonitis in 1. Acid-fast smear results for sputum, bronchoalveoar lavage fluid or peritoneal fluid, or from lymph node samples were positive for 13 of 22 patients with culture positive MTB. The remaining 9 patients had acid-fast smear-negative and culture-positive results for MTB.

The clinical characteristics of 22 patients with active TB are shown in Table 1. Regarding the treatment, all patients received INH, RFP, PZA and EB daily or SM three times a week for the initial two months and INH, RFP, EB daily for the following four months according to the treatment for active TB recommended by WHO. All patients completed the antituberculosis treatment without any remarkable side effects or abnormal laboratory findings. The clinical effects of all patients were good.

The results of QFT-2G test for patients with active TB disease before the initiation of antituberculosis treatment are shown in Table 2. The positive response of QFT-2G test was recognized in $82 \%$, a negative response in $9 \%$, and indeterminate response in $9 \%$.

Transitional changes in QFT-2G test results for patients with active TB are shown in Table 3. The rate of positive QFT-2G test results and IFN- $\gamma$ level of peripheral blood significantly decreased from the level before treatment to the level on completion of treatment. Thereafter, the level 
slightly decreased from that six months after the completion of treatment to the level 36 months later and there were no significant differences between the two sets of data. As the remarkable findings of transitional change of QFT-2G tests results, the positive response of QFT-2G test continued for three years after the initiation of antituberculosis treatment despite the improvement of clinical symptoms or radiological findings of active TB.

The relationship between cut-off values divided into 10 percent increments and conversion rate of QFT-2G test results at the completion of treatment is shown in Table 4. When the cut-off value was below $70 \%$, the conversion rate was $100 \%$ and when the cut-off value was below $60 \%$, the conversion rate decreased to $94 \%$. When the cut-off value was below $50 \%$, the conversion rate decreased to $83 \%$. We also investigated the relationship between cut-off values and conversion rate of QFT-2G test results three years after the initiation of treatment as well as that at the finish of treatment (Table 5). When the cut-off value was below 60\%, the conversion rate was $100 \%$. However, when the cut-off value was below $50 \%$, the conversion rate decreased to $89 \%$.

Table 2. Results of QFT-2G Tests among Patients with Active TB before the Initiation of Antituberculosis Treatment

\begin{tabular}{lc}
\hline QFT-2G test result & $\begin{array}{c}\text { Patients with active TB disease } \\
(\mathrm{n}=22)\end{array}$ \\
\hline Positive & $18(82 \%)$ \\
Negative & $2(9 \%)$ \\
Indeterminate & $2(9 \%)$ \\
\hline Total & $22(100 \%)$ \\
\hline
\end{tabular}

QFT-2G : QuantiFERON TB-2G

Discussion

To our knowledge, this study involves the first cohort investigating the effect of antituberculosis treatment on T-cell responses to MTB-specific antigens on long-term follow-up after the completion of treatment. Subsequently, we found that although the positive response rate for QFT-2G test (for ESAT-6 and/or CFP-10) significantly decreased from $82 \%$ before treatment to $50 \%$ at treatment completion (six months later) and mean IFN- $\gamma$ level was also significantly decreased from $0.75 \mathrm{IU} / \mathrm{mL}$ to $0.39 \mathrm{IU} / \mathrm{mL}$, there were no significant differences in the positive response rates for QFT-2G test or IFN- $\gamma$ level of peripheral blood for a few years after the finish of antituberculosis treatment. Concerning the influence of active TB or LTBI treatment on the Tcell response to MTB-specific antigens, two reports using the ELISPOT method showed declines in T-cell response to ESAT-6 with treatment of active TB in small numbers of patients. Nicol et al reported an early increase in ELISPOT responses to ESAT-6 and CFP-10 followed by a decline after three and six months of active TB treatment in ten children (4). Aiken et al showed declines in ESAT-6 and CFP-10 ELISPOT counts after successful TB treatment 12 months after diagnosis in 89 patients with TB in Gambia (5). In our previous study (6), IGRAs using QFT-2G tests showed a significant transitional decrease between the initiation of antituberculosis treatment and the finish of treatment. Concerning the reason that the IFN- $\gamma$ response was maintained without a decrease after the completion of antituberculosis treatment, we cannot clarify whether the persistence of IFN- $\gamma$ response is dependent upon the T-cell immunity of the individual patient, on the bacterial virulence, or on the cut-off level of QFT-2G test.

In order to resolve the second problem, we challenged the change in conversion criteria from a positive response to a

Table 3. Transitional Change in QFT-2G Test Results for Patients with Active TB During and after Treatment with Antituberculosis Drugs

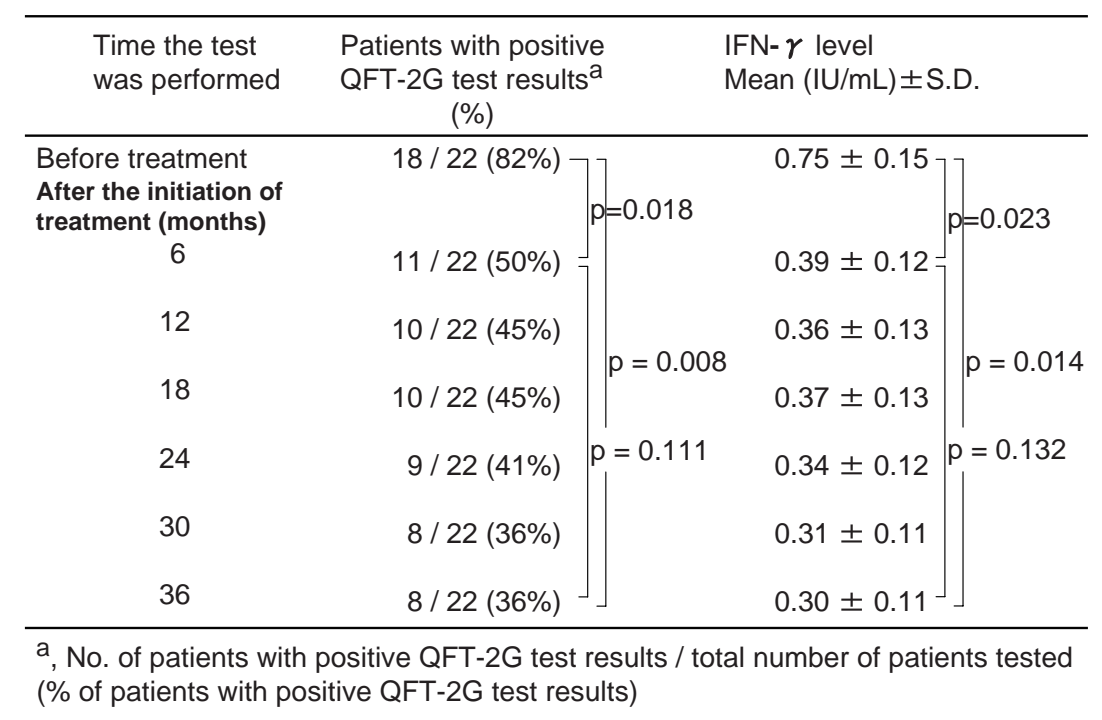


Table 4. Conversion Rate of QFT-2G Test Results at the Completion of Treatment

\begin{tabular}{cc}
\hline Cut-off value (\%) ${ }^{\text {a }}$ & $\begin{array}{c}\text { Conversion rate of QFT-2G } \\
\text { test results }(\%)\end{array}$ \\
\hline $90 \geqq$ & $18 / 18(100)$ \\
$80 \geqq$ & $18 / 18(100)$ \\
$70 \geqq$ & $18 / 18(100)$ \\
$60 \geqq$ & $17 / 18(94)$ \\
$50 \geqq$ & $15 / 18(83)$ \\
$40 \geqq$ & $12 / 18(67)$ \\
$30 \geqq$ & $10 / 18(56)$ \\
$20 \geqq$ & $4 / 18(22)$ \\
$10 \geqq$ & $0 / 18(0)$ \\
\hline
\end{tabular}

a : IFN- $\gamma$ level at the completion of treatment / IFN- $\gamma$ level of baseline peak value (\%)

Table 5. Conversion Rate of QFT-2G Test Results 3 Years after the Initiation of Treatment

\begin{tabular}{cc}
\hline Cut-off value (\%) a & $\begin{array}{c}\text { Conversion rate of } \\
\text { QFT-2G test results (\%) }\end{array}$ \\
$90 \geqq$ & $18 / 18(100)$ \\
$80 \geqq$ & $18 / 18(100)$ \\
$70 \geqq$ & $18 / 18(100)$ \\
$60 \geqq$ & $18 / 18(100)$ \\
$50 \geqq$ & $16 / 18(89)$ \\
$40 \geqq$ & $14 / 18(78)$ \\
$30 \geqq$ & $12 / 18(67)$ \\
$20 \geqq$ & $7 / 18(39)$ \\
$10 \geqq$ & $2 / 18(11)$ \\
\hline a : IFN- $\gamma$ level 3 years after the initiation of treatment / IFN- $\gamma$ level of \\
baseline peak value (\%)
\end{tabular}

negative response of IFN- $\gamma$. Namely, in the interpretation of the IGRAs on serial testing, we defined the cut-off value of a positive response as the ratio of IFN- $\gamma$ mean level at the completion of treatment for IFN- $\gamma$ mean level of baseline peak value divided into 10 percent increments shown in Tables 4,5 . When the cut-off value was below $50 \%$, we could judge the conversion of QFT-2G test in most cases except for two cases $(89 \%)$ three years after the initiation of antituberculosis treatment through this study. Pai et al compared serial testing using the QFT Gold test with the TST test in Indian health care workers (7), and found a stronger agreement using the QFT conversion definition of IFN- $\gamma$ over $0.70 \mathrm{IU} / \mathrm{mL}$, rather that the manufacturer's recommendation of IFN- $\gamma$ over $0.35 \mathrm{IU} / \mathrm{mL}$, with TST conversion (defined as a $10 \mathrm{~mm}$ increment). They reported that QFT nonreverters had significantly higher baseline IFN- $\gamma$ levels than reverters because the IFN- $\gamma$ level in the former had to drop more for the result to become negative. Otherwise, QFT reverters were more likely to have a baseline TST reading less than
$10 \mathrm{~mm}$ compared with that of nonreverters, and they suggested that these baseline positive QFT results may have been false positive. Considering their explanation, we may have to reconsider the cut-off levels of IFN- $\gamma$ assays with regard to positive responses especially in patients with active TB during antituberculosis treatment for several years after the completion of treatment as described in the report by Pai et al (1).

Because there is a small scale study in this report, it is difficult to decide the conversion criteria from a positive response to a negative response of IFN- $\gamma$ on serial testing through this study. Therefore, we would like to undertake a large scale study about the cut-off levels of IFN- $\gamma$ assays with regard to positive responses in patients with active TB during antituberculosis treatment for a long-time after the completion of treatment.

In conclusion, we demonstrated that a high IFN- $\gamma$ level persists for at least a few years after the initial completion of antituberculosis treatment. It has been thought that the 
high cut-off level for a positive response on QFT-2G test caused an increase in the positive response rate of the QFT-
$2 \mathrm{G}$ test. It may be better to reconsider the cut-off level for positive responses in the QFT-2G test.

\section{References}

1. Pai M, Joshi R, Dogra S, et al. Serial testing of health care workers for tuberculosis using interferon- $\gamma$ assay. Am J Respir Crit Care Med 174: 349-355, 2006.

2. Chee CBE, KhinMar KW, Gan SH, Barkham TMS, Pushparani M, Wang YT. Latent tuberculosis infection treatment and T-cell responses to Mycobacterium tuberculosis-specific antigens. Am J Respir Crit Care Med 175: 282-287, 2007.

3. Mazurek GH, Jereb J, Lobue P, Iademarco MF, Mdetchock B, Vernon A. Division of Tuberculosis Elimination, National Center for HIV, STD, and TB Prevention, Centers for Disease Control and Prevention. Guidelines for using the QuantiFERON-TB Gold test for detecting Mycobacterium tuberculosis infection, United States. MMWR Recomm Rep 54: 49-55, 2005.

4. Nicol MP, Pienaar D, Wood K, et al. Enzyme-linked immunospot assay responses to early secretory antigenic target 6 , culture fil- trate protein 10, and purified protein derivative among children with tuberculosis: implication for diagnosis and monitoring of therapy. Clin Infect Dis 40: 1301-1308, 2005.

5. Aiken AM, Hill PC, Fox A, et al. Reversion of ELISPOT test after treatment in Gambian tuberculosis cases. BMC Infect Dis 6: 66, 2006.

6. Kobashi Y, Obase Y, Fukuda M, Yoshida K, Miyashita N, Oka M. Clinical reevaluation of the QuantiFERON TB-2G test as a diagnostic method for differentiating active tuberculosis from nontuberculous mycobacteriosis. Clin Infect Dis 43: 1540-1546, 2006.

7. Pai M, Joshi R, Dogra S, et al. Persistently elevated T-cell interferon-gamma responses after treatment for latent tuberculosis infection among health care workers in India: a preliminary report. J Occup Med Toxicol 1: 7, 2006.

(C) 2008 The Japanese Society of Internal Medicine http://www.naika.or.jp/imindex.html 